\section{Demonstration of intracytoplasmic needle-like inclusions in hepatocytes of patients with porphyria cutanea tarda}

KR JAMES, JM CORTÉS, ${ }^{*}$ AND FJ PARADINAS Charing Cross Hospital and Medical School, London; *Clínica de la Concepción, Universidad Autónoma, Madrid, Spain

Porphyria cutanea tarda (PCT) is a bullous dermatosis associated with liver damage and accumulation of uroporphyrin and heptacarboxyporphyrins in the liver. This accumulation leads to red autofluorescence of unfixed air-dried sections with ultraviolet light ${ }^{1}$ and to the presence of needle-like inclusions in hepatocytes $^{23}$ which are thought to represent crystallised porphyrins and which appear specific for, and therefore diagnostic of, PCT.

There are many reports of the light microscopy of the liver in PCT, and it is suprising that whereas in some of them inclusions were seen in almost every patient, ${ }^{34}$ no mention of them is made in most series. ${ }^{5-8}$ It appeared to us significant that inclusions have been reported to disappear during most staining procedures other than a rapid haematoxylin and eosin, ${ }^{4}$ and we felt that this could be due to their being dissolved by one of the reagents used.

Accordingly, a paraffin block of $10 \%$ formalin fixed liver from a case of PCT was examined for the presence of such inclusions. Sections were cut at $5 \mu \mathrm{m}$, floated briefly on warm water, picked up on slides, and dried at $37^{\circ} \mathrm{C}$ overnight.

One section was dewaxed in xylene and mounted in Ralmount. $\dagger$ Numerous brown, needle-shaped birefringent crystals were clearly visible when examined microscopically. When examined with the fluorescence microscope using BG12 and BG38 exciter filters and a K530 barrier filter, strong red fluorescence broadly confined to the same areas as the crystals was seen. A further section was dewaxed, placed in absolute ethanol for 1 hour, cleared in xylene, and mounted. The results were similar to those of the first section.

A series of sections were next dewaxed and taken down to running tap water. They were left washing for $2,5,10,20,40$, and 60 minutes, then dehydrated, cleared, and mounted. The crystals were totally removed after 10 minutes. The red fluorescence

†RA Lamb, 6, Sunbeam Road, London NW10 6JL, UK.

Received for publication 20 March 1980

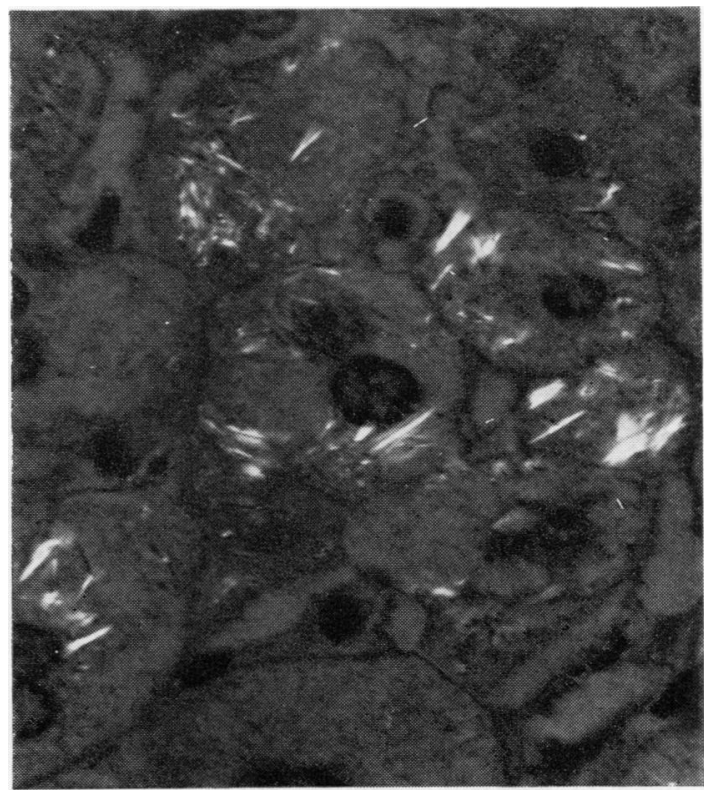

(a)

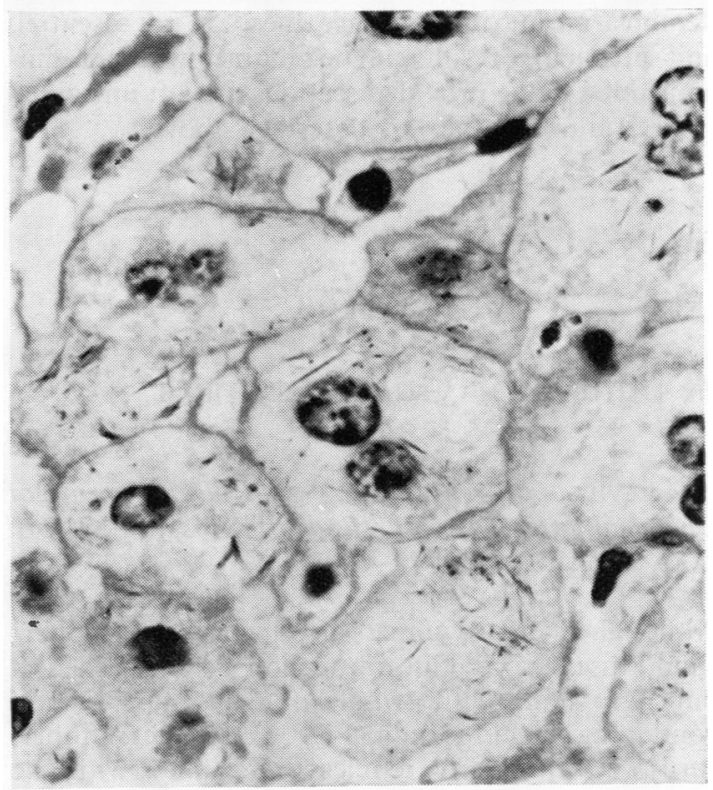

(b)

Acicular (needle-like) intracytoplasmic inclusions viewed $(a)$ with and $(b)$ without polarised light. Modified Cole's haematoxylin (see text) $\times 1000$. 
became weaker but was not completely removed even after 60 minutes.

Lack of any tissue staining was a disadvantage, and a further section was taken to $70 \%$ alcohol and then stained in Cole's haematoxylin ${ }^{9}$ for 30 seconds, rinsed in tap water for 10 seconds, dehydrated, cleared, and mounted. This provided adequate nuclear staining, and the crystals (Figure) and red fluorescence were unaffected. Using this method, inclusions and fluorescence were demonstrated in two other old paraffin blocks of liver tissue from patients with PCT. Neither inclusions nor fluorescence had been observed in the earlier examination of routinely prepared paraffin sections from this material.

It is suggested that failure to demonstrate needlelike inclusions in cases of PCT may be due to the treatment of sections with water. Of interest was the persistence of typical porphyrin autofluorescence in sections from routinely fixed and processed paraffin blocks stored for several years.

It is well known that contact with water must be avoided to preserve optimum autofluorescence in fresh cryostat sections: ${ }^{1}$ prolonged contact with water during paraffin wax processing and slide preparation should also be avoided to preserve the characteristic inclusions of PCT. Although frozen sections of fresh tissue are the method of choice to demonstrate autofluorescence, these are not essential, and satisfactory results can be obtained with paraffinembedded tissue provided sections are left unstained or are stained in the way described above.

\section{A punch for Guthrie papers}

WJ REVILL AND RH WILKINSON Department of Chemical Pathology, John Radcliffe Hospital, Oxford $O X 39 D U, U K$

Although there are numerous punches for removing small discs from Guthrie papers ${ }^{1 *}$ we needed a punch to remove the whole of the square section containing the blood spot (Fig. 3c). On each Guthrie card we require four circles to be filled with the

*See HM (69) 72 para 7 request form/filter paper (form HMR 101/6).

Received for publication 18 March 1980
We are grateful to Professor H Oliva (Universidad Autónoma of Madrid) and Dr B Portmann (Liver Unit, King's College Hospital) for the loan of liver tissue from patients with PCT.

\section{References}

${ }^{1}$ Enerbäck L, Lundvall O. Properties and distribution of liver fluorescence in porphyria cutanea tarda. Virchows Arch 1970;350:293-302.

2 Timme $\mathrm{AH}$. The ultrastructure of the liver in human symptomatic porphyria. $S$ Afr Med J 1971;25:58-62.

${ }^{3}$ Waldo ED, Tobias H. Needle-like cytoplasmic inclusions in the liver in porphyria cutanea tarda. Arch Pathol 1973; 96:368-71.

${ }^{4}$ Cortés JM, Oliva H, Paradinas FJ, Hernandez-Guio C. The pathology of the liver in porphyria cutanea tarda. Histopathology (in press).

${ }^{5}$ Doss M, Look D, Henning H. Chronic hepatic porphyria in chronic aggressive hepatitis. Klin Wochenschr 1971;49: 52-4.

${ }^{6}$ Elder GH, Gray $\mathrm{CH}$, Nicholson DC. The porphyrias: a review. J Clin Pathol 1972;25:1013-33.

${ }^{7}$ Topi GC, D'Alessandro Gandolfo L. Liver in porphyria cutanea tarda. In Porphyrins in Human Diseases. Basel: Karger 1976;312-4.

${ }^{8}$ Meyer UA, Schmid R. In: Stambury JB, Wyngaarden JB, Fredrickson DS, eds. The Porphyrias in the Metabolic Basis of Inherited Disease. McGraw-Hill, 1978;1167-220.

${ }^{9}$ Cole EC. Studies on haematoxylin stains. Stain Technol $1943 ; 18: 125-42$.

Requests for reprints to: Mr KR James, Department of Histopathology, Charing Cross Hospital Medical School, Fulham Palace Road, Hammersmith, London W6 8RF, UK.

blood; a maximum of two are required for the amino acid screen technique ${ }^{2}$ for the detection of excess phenylalanine in phenylketonuria. We added thyroid stimulating hormone (TSH) estimation to the screen for the diagnosis of hypothyroidism in the newborn. The case number of the patient is written on each of the last two squares. They are punched from the Guthrie card and sent to the regional assay laboratory for TSH estimation. This punch speeds up the preparation of the squares.

\section{The machine}

The punch consists of a frame of aluminium alloy (Fig. 1). Above the frame is a perforated table with a backstop. The table is raised above the base plate 